\section{(อ) OPEN ACCESS}

\title{
Antimicrobial resistance in Mycoplasma genitalium sampled from the British general population
}

\author{
Rachel Pitt, ${ }^{1}$ Magnus Unemo, ${ }^{2}$ Pam Sonnenberg, ${ }^{3}$ Sarah Alexander 이, \\ Simon Beddows, ${ }^{1}$ Michelle Jayne Cole ${ }^{0},{ }^{1}$ Soazig Clifton, ${ }^{3}$ Catherine $\mathrm{H}$ Mercer, ${ }^{3}$ \\ Anne M Johnson, ${ }^{4}$ Catherine A Ison, ${ }^{1}$ Nigel Field ${ }^{4}{ }^{4}$
}

\begin{abstract}
${ }^{1}$ National Infection Service, Public Health England, London, United Kingdom

${ }^{2}$ WHO Collaborating Centre for Gonorrhoea and Other STIs, National Reference Laboratory for STIS, Department of Laboratory Medicine, Microbiology, Faculty of Medicine and Health, Örebro University, Orebro, Sweden ${ }^{3}$ Centre for Population Research in Sexual Health and HIV, Institute for Global Health, UCL, London, United Kingdom ${ }^{4}$ Centre for Molecular Epidemiology and Translational Research, Institute for Global Health, UCL, London, United Kingdom
\end{abstract}

\section{Correspondence to}

Dr Nigel Field, Centre for Molecular Epidemiology and Translational Research, Institute for Global Health, UCL, London WC1E 6JB, UK; nigel.field@ucl. ac.uk

Received 15 May 2019 Revised 20 November 2019 Accepted 12 December 2019 Published Online First 10 January 2020
Check for updates

(c) Author(s) (or their employer(s)) 2020. Re-use permitted under CC BY. Published by BMJ.

To cite: Pitt $R$, Unemo $M$, Sonnenberg P, et al. Sex Transm Infect

2020:96:464-468.

\section{ABSTRACT \\ Background Mycoplasma genitalium is a common} sexually transmitted infection. Treatment guidelines focus on those with symptoms and sexual contacts, generally with regimens including doxycycline and/or azithromycin as first-line and moxifloxacin as second-line treatment. We investigated the prevalence of antimicrobial resistance (AMR)-conferring mutations in $M$. genitalium among the sexually-active British general population. Methods The third national survey of sexual attitudes and lifestyles (Natsal-3) is a probability sample survey of 15162 men and women aged 16-74 years in Britain conducted during 2010-12. Urine test results for $M$. genitalium were available for 4507 participants aged 16-44 years reporting $\geq 1$ lifetime sexual partner. In this study, we sequenced regions of the 23S rRNA and parC genes to detect known genotypic determinants for resistance to macrolides and fluoroquinolones respectively.

Results $94 \%(66 / 70)$ of specimens were re-confirmed as $M$. genitalium positive, with successful sequencing in $85 \%(56 / 66)$ for $23 S$ rRNA and $92 \%(61 / 66)$ for parC genes. Mutations in 23S rRNA gene (position A2058/A2059) were detected in 16.1\% (95\% Cl: $8.6 \%$ to $27.8 \%$ ) and in parC (encoding ParC D87N/ D87Y) in 3.3\% (0.9\%-11.2\%). Macrolide resistance was more likely in participants reporting STI diagnoses (past 5 years) $(44.4 \%(18.9 \%-73.3 \%)$ vs $10.6 \%$ $(4.6 \%-22.6 \%) ; p=0.029)$ or sexual health clinic attendance (past year) $(43.8 \%(23.1 \%-66.8 \%)$ vs $5.0 \%$ $(1.4 \%-16.5 \%) ; p=0.001)$. All 11 participants with AMRconferring mutations had attended sexual health clinics (past 5 years), but none reported recent symptoms. Conclusions This study highlights challenges in M. genitalium management and control. Macrolide resistance was present in one in six specimens from the general population in 2010-2012, but no participants with AMR M. genitalium reported symptoms. Given anticipated increases in diagnostic testing, new strategies including novel antimicrobials, AMR-guided therapy, and surveillance of AMR and treatment failure are recommended.

\section{INTRODUCTION}

Mycoplasma genitalium is a widespread STI causing urogenital infection in men and women. ${ }^{1-3} M$. genitalium infection is a frequent cause of nongonococcal urethritis in men, and is associated with cervicitis, pelvic inflammatory disease, preterm delivery, spontaneous abortion, and infertility in women. ${ }^{2-6}$ Most individuals with M. genitalium are asymptomatic, and the clinical implications of symptomless infection remain unclear.

In the British general population, in 20102012, the urogenital prevalence of M. genitalium was similar to that of Chlamydia trachomatis in those aged 16-44 years old, although co-infection was rare. In men, $M$. genitalium prevalence was $1.2 \%$ (95\% CI $0.7 \%$ to $1.8 \%$ ) compared with C. trachomatis prevalence of $1.1 \%(0.7 \%-1.6 \%)$, and in women was $1.3 \%(0.9 \%-1.9 \%)$ compared with $1.5 \%(1.1 \%-2.0 \%)$ respectively. ${ }^{78}$ Higher M. genitalium prevalence has been observed in men who have sex with men (MSM) (3.2\% (2.1\%-5.1\%)) and female sex workers (15.9\% (13.5\%-18.9\%)). ${ }^{1}$

National guidelines for M. genitalium are lacking or only recently introduced for many countries. Existing international and national guidelines, including the 2018 UK national guidelines, focus on testing those with symptoms and sexual contacts of cases, citing a lack of evidence to recommend routine screening in asymptomatic individuals, which might have implications for selection of antimicrobial resistance (AMR). ${ }^{9-11}$ Due to suboptimal diagnostics and a lack of testing even within specialist centres, M. genitalium is likely to be widely underdiagnosed.

The fastidious nature and slow growth of the organism makes culture challenging, timeconsuming, and unsuitable for routine diagnostic testing. Accordingly, routine AMR phenotyping of M. genitalium is not feasible, and AMR-testing currently relies on the detection of genotypic changes within genomic loci associated with phenotypic resistance and treatment failure. Current treatment guidelines typically recommend a macrolide (azithromycin) or tetracycline (doxycycline) followed by azithromycin, ideally after AMR testing, as first line and a fluoroquinolone (moxifloxacin) as second line treatment. ${ }^{9-11}$ However, azithromycin resistance (5\%-100\%) and moxifloxacin resistance $(0 \%-30 \%)$ have been reported in studies from Europe, Australia, New Zealand, Japan, and USA. ${ }^{12-15}$ These data derive from clinicbased studies, and there are currently no data on the occurrence of M. genitalium AMR determinants in specimens from the general population to inform policy decisions. 
Taken together, M. genitalium is an important and prevalent STI, and one where management and control is challenging because it is under-tested, under-detected, and difficult to treat. ${ }^{16}$ Moreover, increasing availability of diagnostic tests is likely to increase opportunities for screening asymptomatic patients, but the risks and benefits need careful consideration. ${ }^{17}$ This situation requires a considered strategic public health response. We investigated the distribution of genotypically determined resistance in M. genitalium positive specimens from the sexually-active British general population.

\section{METHODS}

\section{Participants and survey procedures}

The third National Survey of Sexual Attitudes and Lifestyles (Natsal-3) was a stratified probability sample survey of 15162 men and women in Britain (England, Scotland, and Wales) aged 16-74 years who were interviewed in 2010-2012. The estimated overall response rate was $57.7 \%$ and the cooperation rate was $65.8 \%$ (of all eligible addresses contacted). ${ }^{18}$ Participants were interviewed using computer-assisted face-to-face and selfcompletion interviews (CASI); further methodological details have been described elsewhere. ${ }^{19}$ The CASI included questions about participants' sexual behaviour, sexual health clinic attendance, current STI symptoms, and history of being diagnosed with STIs by a healthcare professional. After the interview, we invited a sample of participants aged $16-44$ years to provide urine for STI testing. ${ }^{8}$ First-void urine $(4-5 \mathrm{~mL})$ was collected with the FirstBurst device and posted to Public Health England for testing. All participants were provided with information on where to obtain free diagnostic STI/HIV testing and sexual health advice. ${ }^{19}$

\section{Laboratory methods}

Urine specimens were tested using an in-house real-time PCR (RT-PCR) assay which targets the M. genitalium adhesin protein $(\mathrm{MgPa})$ gene (assay modified from Jensen et al. 2004), ${ }^{20}$ with positive or equivocal results confirmed using a research-use-only Aptima M. genitalium assay (Hologic Inc., San Diego, USA). Urine test results were available for M. genitalium on $4507 \mathrm{spec}-$ imens and 72 were positive. For specimens where participants had provided consent for storage (70/72), the presence of $M$. genitalium DNA was re-confirmed in the present study using the in-house $\mathrm{MgPa}$ RT-PCR. ${ }^{20}$ Macrolide and fluoroquinolone resistance determinants were assessed by PCR and sequencing of resistance-associated regions of the $23 \mathrm{~S}$ rRNA and parC gene and specimens were attributed a resistant or susceptible (wildtype sequence or synonymous mutations) genotype. ${ }^{21-23}$

\section{Statistical analysis}

We undertook a descriptive statistical analysis to examine the proportion of M. genitalium cases with genetic determinants of AMR and the participant characteristics associated with AMR. Exact 95\% confidence intervals were calculated, and proportions compared for specimens with genetic determinants of macrolide resistance by sociodemographic, behavioural, and clinical characteristics using two-sided Fisher's exact tests.

\section{RESULTS}

Of the available specimens that previously tested positive for $M$. genitalium, 94\% (66/70) were re-confirmed as positive for $M$. genitalium. Sequencing was successful in $85 \%(56 / 66)$ for $23 \mathrm{~S}$ rRNA and 92\% (61/66) for parC genes.
Table 1 Wild-Type and antimicrobial resistance-conferring mutations in 23S rRNA and parC genes in Mycoplasma genitalium positive specimens from the general British population

\begin{tabular}{lll}
\hline 23S rRNA gene & No. ( $\mathbf{n = 5 6 )}$ & \% (95\% confidence intervals) \\
\hline Wild-type (S) & 47 & 83.9 (72.2 to 91.3$)$ \\
\hline Mutation detected (R) & 9 & 16.1 (8.6 to 27.8) \\
A2058G & 7 & \\
A2059C & 1 & \\
A2059G & 1 & \\
\hline parC gene & No. ( $\mathbf{n}=61)$ & $\%$ (95\% confidence intervals) \\
\hline Wild-type (S) & 59 & 96.7 (88.9 to 99.1) \\
\hline Mutation detected (R) & 2 & $3.3(0.9$ to 11.2$)$ \\
D87N & 1 & \\
D87Y & 1 & \\
\hline
\end{tabular}

*includes isolates with wild-type sequence $(n=56)$ and synonymous mutations $(n=4)$.

$\mathrm{R}$, resistant; S, susceptible.

Mutations associated with macrolide resistance, at nucleotide position 2058 or 2059 of the 23S rRNA gene (Escherichia coli numbering), were detected in $9 / 56(16.1 \%$; 95\% CI $8.6 \%$ to $27.8 \%$ ) specimens, with the A2058G mutation most common $(n=7)$, followed by A2059G $(n=1)$ and A2059C $(n=1)($ table 1$)$. parC gene mutations associated with fluoroquinolone resistance (D87N and D87Y $)^{2324}$ were detected in 2/61 (3.3\%; 0.9\%$11.2 \%)$ specimens. For these two specimens, the $23 \mathrm{~S}$ rRNA gene sequence was wild-type $(n=1)$ or did not amplify $(n=1)$.

Specimens with mutations associated with macrolide resistance were more likely to come from participants reporting a history of diagnosed STIs or sexual health clinic attendance (table 2). Participants reporting any STI diagnoses in the past 5 years were more likely to have macrolide resistant $M$. genitalium than those without a STI diagnosis (44.4\% (18.9\%-73.3\%) vs $10.6 \%(4.6 \%-22.6 \%) ; \mathrm{p}=0.029)$. Similarly, those reporting visiting a sexual health clinic in the past year were more likely to have macrolide resistant $M$. genitalium than those not attending a clinic in the past year $(43.8 \%(23.1 \%-66.8 \%)$ vs $5.0 \%$ $(1.4 \%-16.5 \%) ; p=0.001)$. A higher proportion of participants reporting two or more sexual partners in the past year had $M$. genitalium with genotypic macrolide resistance when compared with participants reporting zero or one partner $(25.0 \%(13.3 \%$ $42.1 \%)$ vs $4.2 \%(0.7 \%-20.3 \%) ; p=0.063)$.

All four participants with macrolide-resistant specimens and STI diagnoses (table 2) had been diagnosed with C. trachomatis in the preceding 5 years, two of whom had Neisseria gonorrhoeae, genital warts and non-specific urethritis (NSU), and one of whom had Treponema pallidum. Of the participants with macrolide-susceptible isolates and STI diagnoses $(n=5)($ table 2), three had been diagnosed with C. trachomatis, one with genital warts and one with Trichomonas vaginalis.

We observed that none of 11 participants with $M$. genitalium AMR mutations (in 23 rRNA or parC genes) reported any symptoms in the past month. Furthermore, these 11 participants all reported sexual health clinic attendance in the past 5 years (seven within the past year). Small numbers prevented further epidemiological characterisation of specimens with mutations in the parC gene.

\section{DISCUSSION}

To our knowledge, this is the first probability sample study representative of the general population to assess the prevalence 
Table 2 Sociodemographic, behavioural, and clinical risk factors for macrolide resistance-conferring mutations in the 235 rRNA gene in Mycoplasma genitalium specimens from a sexually-active probability sample of the general British population

\begin{tabular}{|c|c|c|c|c|c|c|c|c|}
\hline & \multicolumn{3}{|c|}{ M. genitalium with resistance-conferring mutation } & \multicolumn{3}{|c|}{ Susceptible $M$. genitalium } & \multirow{2}{*}{$\begin{array}{l}\text { Total } \\
\text { No. }\end{array}$} & \multirow[b]{2}{*}{$P$ value } \\
\hline & No. & $\%$ & $95 \% \mathrm{Cl}$ & No & & $95 \% \mathrm{Cl}$ & & \\
\hline & 9 & 16.1 & 8.7 to 27.8 & 47 & 83.9 & 72.2 to 91.3 & 56 & \\
\hline \multicolumn{9}{|l|}{ Sex } \\
\hline Male & 3 & 14.3 & 5.0 to 34.6 & 18 & 85.7 & 65.4 to 95.0 & 21 & 1.000 \\
\hline Female & 6 & 17.1 & 8.1 to 32.7 & 29 & 82.9 & 67.3 to 91.9 & 35 & \\
\hline \multicolumn{9}{|l|}{ Age (years) } \\
\hline $16-24$ & 5 & 22.7 & 10.1 to 43.4 & 17 & 77.3 & 56.6 to 89.9 & 22 & 0.479 \\
\hline $25-34$ & 4 & 14.8 & 5.9 to 32.5 & 23 & 85.2 & 67.5 to 94.1 & 27 & \\
\hline $35-44$ & 0 & 0 & - & 7 & 100.0 & 64.6 to 100 & 7 & \\
\hline \multicolumn{9}{|l|}{ Ethnic group* } \\
\hline White & 7 & 15.6 & 7.8 to 28.8 & 38 & 84.4 & 71.2 to 92.3 & 45 & 0.611 \\
\hline Black/Black British & 2 & 25.0 & 7.2 to 59.1 & 6 & 75.0 & 40.9 to 92.9 & 8 & \\
\hline \multicolumn{9}{|c|}{ No. sexual partners, past yeart } \\
\hline $2+$ & 8 & 25.0 & 13.3 to 42.1 & 24 & 75.0 & 57.9 to 86.8 & 32 & 0.063 \\
\hline $0-1$ & 1 & 4.2 & 0.7 to 20.3 & 23 & 95.8 & 79.8 to 99.3 & 24 & \\
\hline \multicolumn{9}{|c|}{ No. new sexual partners, past year $\uparrow$} \\
\hline $1+$ & 8 & 24.2 & 12.8 to 41.0 & 25 & 75.7 & 59.0 to 87.2 & 33 & 0.067 \\
\hline 0 & 1 & 4.4 & 0.8 to 21.0 & 22 & 95.7 & 79.0 to 99.2 & 23 & \\
\hline \multicolumn{9}{|l|}{ Unsafe sex, past year $¥$} \\
\hline Yes & 3 & 21.4 & 7.6 to 47.6 & 11 & 78.6 & 52.4 to 92.4 & 14 & 0.678 \\
\hline No & 6 & 14.6 & 6.9 to 28.4 & 35 & 85.4 & 72.6 to 93.1 & 41 & \\
\hline \multicolumn{9}{|c|}{ STI symptoms, past month§ } \\
\hline Yes & 0 & 0 & - & 12 & 100.0 & 75.8 to 100 & 12 & 0.180 \\
\hline No/not mentioned & 9 & 20.5 & 11.2 to 34.5 & 35 & 79.5 & 65.5 to 88.9 & 44 & \\
\hline \multicolumn{9}{|c|}{ Diagnosed with any STI, past 5 years } \\
\hline Yes & 4 & 44.4 & 18.9 to 73.3 & 5 & 55.6 & 26.7 to 81.1 & 9 & 0.029 \\
\hline No & 5 & 10.6 & 4.6 to 22.6 & 42 & 89.4 & 77.4 to 95.4 & 47 & \\
\hline \multicolumn{9}{|c|}{ Sexual health clinic attendance } \\
\hline Yes & 9 & 24.3 & 13.4 to 40.1 & 28 & 75.7 & 59.9 to 86.6 & 37 & 0.021 \\
\hline In the last year & 7 & 43.8 & 23.1 to 66.8 & 9 & 56.3 & 33.2 to 76.9 & 16 & 0.001 \\
\hline 1+years ago & 2 & 12.5 & 3.5 to 36.0 & 19 & 90.5 & 71.1 to 97.4 & 21 & \\
\hline No & 0 & 0 & - & 19 & 100.0 & 83.2 to 100 & 19 & \\
\hline
\end{tabular}

${ }^{*}$ Small numbers of participants in other ethnic groups prevented analysis.

†ncludes both opposite-sex and same-sex partners.

¥Had sex with at least two partners in the past year and did not use a condom during this timeframe; one participant did not respond to this question.

$\S S T I$ symptoms included: pain or increased frequency of urination, presence of genital warts or ulcers, penile discharge or abnormal/odorous vaginal discharge, painful testicles, vaginal pain during sex, bleeding between periods or after sex and lower abdominal/pelvic pain.

of mutations associated with AMR in M. genitalium specimens at a national level. Among M. genitalium positive specimens collected in 2010-12, we observed genetic determinants of macrolide resistance in one in six $(16.1 \%)$ specimens and determinants of fluoroquinolone resistance in one in thirty $(3.3 \%)$. Specimens with macrolide resistance were more likely to come from participants reporting a history of diagnosed bacterial STIs or previous clinic attendance. It was not possible to determine whether the observed prevalence of AMR is attributable to sexual transmission of resistant M. genitalium strains or the emergence of de novo resistance.

Study strengths include the high proportion of specimens with sequencing data and the collection of specimens from the general population, which is important because previous studies of $M$. genitalium AMR have mainly used convenience samples, often drawn from clinic or STI-diagnosed populations, where selection bias may lead to overestimating the scale of the problem. One clinical study collected data at a similar time to Natsal-3 (2010-12) in the UK and provides relevant data for comparison. Pond et al undertook an observational study to assess the proportion with genetic AMR determinants in men presenting with urethritis to a London sexual health clinic in 2011. Nine of $22(41 \%)$ cases with $M$. genitalium had 23S rRNA gene mutations associated with macrolide resistance, and one case had a parC mutation associated with fluoroquinolone resistance. As might be expected, compared with this clinical study, the prevalence with resistance detected was lower in specimens collected from the general population in Natsal. Our study also provides an opportunity to assess AMR in symptomatic and asymptomatic people with M. genitalium in the general population. We expect the high levels of AMR found in Britain to have increased since the specimens were collected given high levels reported for other countries at later times, and we will update findings for the UK in 2022 through the Natsal-4 study. ${ }^{25}$

Potential limitations include that specimens were stored for up to 6 years before re-confirmation and AMR testing, with the potential for specimen degradation, which may explain instances of assay failure. Numerous mutations have been reported in the parC gene highlighting the variability of this region, and we have inferred phenotype from genotype. However, in the present study we only report on $23 \mathrm{~S}$ rRNA or parC gene mutations that have been associated with azithromycin or moxifloxacin 
resistance. The challenge for the microbiological community will be to improve culture capacity and sequencing directly from clinical specimens to better understand how other $23 \mathrm{~S}$ rRNA gene or parC mutations are associated with genotype, phenotype and treatment failures. Furthermore, widely implementing test of cure, genetic AMR testing and surveillance of treatment failures will improve understanding of the correlation between genotype and clinical outcome. Finally, small numbers of M. genitalium positive specimens, even within such a large study, meant there was not sufficient statistical power to rule out associations being present where none were observed.

It was striking that none of the specimens with an AMRconferring genotype were from participants reporting STI symptoms. Under the current UK treatment guidelines, asymptomatic patients are not recommended for M. genitalium testing except sexual contacts, and it is unlikely that any of these participants would have been tested. We need better understanding about the implications of asymptomatic infection, which might resolve with limited sequelae. The current approach might need rethinking if asymptomatic infections are found to be an important reservoir for AMR and/or a source of infection and disease. Given that all participants with AMR specimens had attended clinics in the past 5 years (most in the last year), modelling studies might be undertaken to investigate the cost effectiveness of routinely testing asymptomatic sexual health clinic attendees (ie, clinicbased screening) for M. genitalium and whether this approach might assist in the control of M. genitalium and associated AMR.

In conclusion, our data support M. genitalium detection strategies that include pre-treatment macrolide resistance testing to guide therapy, with the use of moxifloxacin treatment where macrolide resistance is identified. We recognise that availability of commercial validated and quality-assured assays for macrolide resistance detection may vary (and there are currently no commercial assays for detection of fluoroquinolone resistance), and even diagnostic testing is not yet widely obtainable in most countries. However, a promising approach has been described in Australia, where doxycycline therapy was followed by macrolide resistance testing to guide treatment. In this study, where azithromycin was given only to macrolide-susceptible cases and sitafloxacin therapy was used for macrolide-resistant cases, $>92 \%$ of M. genitalium infections were shown to be cured..$^{26}$ Our data highlight the significant public health challenges in the control of M. genitalium, including an urgent need for evidence-based third line treatments, such as pristinamycin, minocycline or new antimicrobials such as lefamulin, gepotidacin, solithromycin or

\section{Key messages}

- We investigated the prevalence of antimicrobial resistance (AMR)-conferring mutations in M. genitalium among the sexually-active British general population

- Macrolide resistance-conferring mutations were detected in $16.1 \%(95 \% \mathrm{Cl}: 8.6 \%$ to $27.8 \%)$ and fluoroquinolone resistance-conferring mutations were detected in $3.3 \%$ $(0.9 \%-11.2 \%)$ of specimens with M. genitalium detected

- Specimens with macrolide resistance were more likely to come from participants reporting a history of diagnosed bacterial STIs or recent sexual health clinic attendance.

- All participants with AMR-conferring mutations had attended sexual health clinics, but none reported recent symptoms

- These data highlight challenges in M. genitalium management and control zoliflodacin, ${ }^{27-30}$ and the need for systematic national and international surveillance of AMR and treatment failure to inform treatment guidelines.

\section{Handling editor Nicola Low}

Twitter Sarah Alexander @sarah1alexander, Soazig Clifton @soazigclifton and Nigel Field @fienige

Acknowledgements We thank: the study participants, the team of interviewers from NatCen Social Research who carried out the interviews, and operations and computing staff from NatCen Social Research; Chinelo Obi, Rebecca Howell-Jones, David Mesher, Heather Northend, Krishna Gupta and Tracey Cairns (Department of HIV and Sexually Transmitted Infections, PHE) for data linkage, anonymization, and data entry; and Kavita Panwar (Virus Reference Department, PHE) for assisting with laboratory testing.

Contributors RP, PS, $\mathrm{Cl}$, and NF conceived this article. RP wrote the first draft with further contributions from all authors. RP did the statistical analysis. PS, CM, AJ, and $\mathrm{Cl}$ were initial applicants on Natsal-3, wrote the study protocol and obtained funding. PS, SC, CM, AJ, Cl, and NF designed the Natsal-3 questionnaire, applied for ethics approval and undertook piloting of the questionnaire. RP, SC, and CM managed data. RP, SA, SB, MC, and CI were responsible for laboratory testing. All authors interpreted data, reviewed successive drafts and approved the final version of the article.

Funding The study was supported by grants from the Medical Research Council (G0701757) and the Wellcome Trust (084840), with contributions from the Economic and Social Research Council and Department of Health.

Competing interests None declared.

\section{Patient consent for publication Not required.}

Ethics approval We obtained ethical approval from Oxfordshire Research Ethics Committee A (09/H0604/27). Participants gave written informed consent to anonymised testing, without the return of results, the ethical rationale for which has been previously described.

Provenance and peer review Not commissioned; externally peer reviewed.

Data availability statement An anonymised Natsal-3 data set has been deposited with the UK Data Service, persistent identified: 10.5255/UKDA-

SN-7799-1. Researchers are also directed to the Natsal website for further information (http://www.natsal.ac.uk).

Open access This is an open access article distributed in accordance with the Creative Commons Attribution 4.0 Unported (CC BY 4.0) license, which permits others to copy, redistribute, remix, transform and build upon this work for any purpose, provided the original work is properly cited, a link to the licence is given, and indication of whether changes were made. See: https://creativecommons.org/ licenses/by/4.01.

\section{ORCID iDs}

Sarah Alexander http://orcid.org/0000-0002-9086-9222

Michelle Jayne Cole http://orcid.org/0000-0002-6707-6910

Nigel Field http://orcid.org/0000-0002-2825-6652

\section{REFERENCES}

1 Baumann L, Cina M, Egli-Gany D, et al. Prevalence of Mycoplasma genitalium in different population groups: systematic review andmeta-analysis. Sex Transm Infect 2018;94:255-62

2 Taylor-Robinson D, Jensen JS. Mycoplasma genitalium: from Chrysalis to Multicolored butterfly. Clin Microbiol Rev 2011;24:498-514.

3 Lis R, Rowhani-Rahbar A, Manhart LE. Mycoplasma genitalium infection and female reproductive tract disease: a meta-analysis. Clin Infect Dis 2015;61:418-26.

4 Manhart LE, Broad JM, Golden MR. Mycoplasma genitalium: should we treat and how? Clin Infect Dis 2011;53:S129-42.

5 Horner PJ, Taylor-Robinson D. Association of Mycoplasma genitalium with balanoposthitis in men with non-gonococcal urethritis. Sex Transm Infect 2011;87:38-40.

6 Soni S, Alexander S, Verlander N, et al. The prevalence of urethral and recta Mycoplasma genitalium and its associations in men who have sex with men attending a genitourinary medicine clinic. Sex Transm Infect 2010;86:21-4.

7 Sonnenberg P, Ison CA, Clifton S, et al. Epidemiology of Mycoplasma genitalium in British men and women aged 16-44 years: evidence from the third National Survey of Sexual Attitudes and Lifestyles (Natsal-3). Int J Epidemiol 2015;44:1982-94.

8 Sonnenberg P, Clifton S, Beddows S, et al. Prevalence, risk factors, and uptake of interventions for sexually transmitted infections in Britain: findings from the National surveys of sexual attitudes and lifestyles (Natsal). The Lancet 2013:382:1795-806.

9 Soni S, Horner P, Rayment M, et al. 2018 BASHH UK national guidleine for the management of infection with Mycoplasma genitalium 2018. 
10 Workowski KA, Bolan G. Sexually Transmitted Diseases Treatment Guidelines, 2015. Centers for Disease Control Morbidity and Mortality Weekly Report(MMWR) 2015:1-137.

11 Jensen JS, Cusini M, Gomberg M, et al. 2016 European guideline on Mycoplasma genitalium infections. J Eur Acad Dermatol Venereol 2016;30:1650-6.

12 Getman D, Jiang A, O'Donnell M, et al. Mycoplasma genitalium prevalence, coinfection, and macrolide antibiotic resistance frequency in a multicenter clinical study cohort in the United States. J Clin Microbio/ 2016;54:2278-83.

13 Gratrix J, Plitt S, Turnbull L, et al. Prevalence and antibiotic resistance of Mycoplasma genitalium among STI clinic attendees in Western Canada: a cross-sectional analysis. BMJ Open 2017;7:e016300.

14 Tagg KA, Jeoffreys NJ, Couldwell DL, et al. Fluoroquinolone and macrolide resistanceassociated mutations in Mycoplasma genitalium. J Clin Microbiol 2013;51:2245-9.

15 Pond MJ, Nori AV, Witney AA, et al. High prevalence of antibiotic-resistant Mycoplasma genitalium in nongonococcal urethritis: the need for routine testing and the inadequacy of current treatment options. Clin Infect Dis 2014;58:631-7.

16 Birger R, Saunders J, Estcourt C, et al. Should we screen for the sexually-transmitted infection Mycoplasma genitalium? evidence synthesis using a transmission-dynamic model. Sci Rep 2017;7:16162.

17 Hughes G, Saunders J. Mycoplasma genitalium: the next sexually transmitted superbug? BMJ 2018;363:k4376.

18 The Amercian Association for Public Opinion Research. Standard definitions: final dispositions of case codes and outcome rates for surveys. 9th edn, 2016.

19 Erens B, Phelps A, Clifton S, et al. Methodology of the third British national survey of sexual attitudes and lifestyles (Natsal-3). Sex Transm Infect 2014;90:84-9.

20 Jensen JS, Bjornelius E, Dohn B, et al. Use of TaqMan 5' nuclease real-time PCR for quantitative detection of Mycoplasma genitalium DNA in males with and without urethritis who were attendees at a sexually transmitted disease clinic. J Clin Microbiol 2004;42:683-92.
21 Pitt $\mathrm{R}$, Fifer $\mathrm{H}$, Woodford $\mathrm{N}$, et al. Detection of markers predictive of macrolide and fluoroquinolone resistance in Mycoplasma genitalium from patients attending sexual health services in England. Sex Transm Infect 2018;94:9-13.

22 Li X, Atkinson TP, Hagood J, et al. Emerging macrolide resistance in Mycoplasma pneumoniae in children: detection and characterization of resistant isolates. Pediatr Infect Dis J 2009;28:693-6.

23 Deguchi T, Maeda S, Tamaki M, et al. Analysis of the gyrA and parC genes of Mycoplasma genitalium detected in first-pass urine of men with non-gonococcal urethritis before and after fluoroquinolone treatment. J Antimicrob Chemother 2001:48:742-4.

24 Gesink D, Racey CS, Seah C, et al. Mycoplasma genitalium in Toronto, ONT: estimates of prevalence and macrolide resistance. Can Fam Physician 2016;62:e96-101.

25 Mercer $\mathrm{CH}$, Clifton S, Prior G, et al. Collecting and exploiting data to understand a nation's sexual health needs: Implications for the British National Surveys of Sexual Attitudes and Lifestyles (Natsal). Sex Transm Infect 2019;95:159-61.

26 Read TRH, Fairley CK, Murray GL, et al. Outcomes of Resistance-guided Sequential Treatment of Mycoplasma genitalium Infections: A Prospective Evaluation. Clin Infect Dis 2019;68:554-60.

27 Paukner S, Gruss A, Jensen JS. In Vitro Activity of Lefamulin against Sexually Transmitted Bacterial Pathogens. Antimicrob Agents Chemother 2018;62. doi:10.1128/AAC.02380-17

28 Jensen JS, Fernandes P, Unemo M. In vitro activity of the new fluoroketolide solithromycin (CEM-101) against macrolide-resistant and -susceptible Mycoplasma genitalium strains. Antimicrob Agents Chemother 2014:58:3151-6.

29 Damião Gouveia AC, Unemo M, Jensen JS. In vitro activity of zoliflodacin (ETX0914) against macrolide-resistant, fluoroquinolone-resistant and antimicrobial-susceptible Mycoplasma genitalium strains. J Antimicrob Chemother 2018;73:1291-4.

30 Jensen JS. P664 in vitro evaluation of gepotidacin, an oral antimicrobial against multidrug-resistant Mycoplasma genitalium. Sex Transm Infect 2019;95(supp 1:A292. 